\title{
Interfacial Layer Effects on the Enhancement Factor of Local Field and Optical Induced Bistability in Small Spherical Metal/Dielectric Composites
}

\author{
Sorry Abdissa , Getnet Melese Meheretu, Sisay Shewamare Gebremichael* \\ College of Natural Science, Jimma University / Wolkite University, Ethiopia
}

Copyright (C) 2015 Horizon Research Publishing All rights reserved.

\begin{abstract}
We studied the interfacial layer effects on the enhancement factor of local field and the optical induced bistability for small spherical metal/dielectric in linear host matrices. It is calculated the interfacial layer effect analytically and numerically on the enhancement factor of local field and the cubic equation of the optical induced bistability of the composite material. We have shown the parameters of the interfacial layer and calculated. We take positive, zero, and negative values which represents dielectric like, no interfacial, and metal like, respectively for the pure metal case. In the case of metal/dielectric for which we consider the interfacial layer particle the above mentioned properties are reversed so that it will be positive to realize the metal like properties of the interfacial layer. The analytical and numerical results show that the enhancement factor of local field is extremely enhanced and the optical induced bistability increased its domain.
\end{abstract}

Keywords Dielectric Function, Interfacial Layer, Enhancement Factor, Optical Bistability

\section{Introduction}

The interfacial layer with the linear and nonlinear response of the medium strongly effects on the enhancement factor of local field and Optical Induced Bistability in the optical material and may even result in the permanent modification of its physical properties. Here, we analyzed the linear and nonlinear optical features of composite materials with metal nanostructures that are dominated by surface plasma oscillations and the interfacial layer as well as the contribution due to the metallic and dielectric properties of the composite material. It is well known that the properties of the surface Plasmon (SP) strongly depends on size, shape, distribution of metal nano-particles and the surrounding dielectric. Composite materials consisting of small nonlinear metallic particles in the shape of sphere which are randomly embedded in a linear dielectric host are well known for their complex responses to incident light fields $[1,2]$.

The studies of the effective optical properties of composite materials were first concerned with the analysis of linear media [3]. The contribution of the local field of the particle was extended studying the nonlinear properties of composite materials $[4,5]$ with the contributions being treated as purely real and small perturbations which can be assumed to be resulting in linear behavior $[5,6]$. Due to these limitations, in the case of relatively high concentration of the nonlinear components, these approximations are generally unable to properly predict the existence optical properties such as the optical bistability associated with the nonlinear optical response of composite materials $[2,4]$. In this paper, we are interested on the nonlinear characteristics of composite materials by considering the interfaces that separates dielectric core from concentric metallic particles embedded in linear dielectric host matrix. It is shown that the interfacial effect plays an important role on the enhancement factor of local field and the optical induced bistability (OIB) of such materials by use of Laplace equation and the classical Drude model. The interfacial factor $I$ is introduced to characterize interfacial layer [6] and has been developed to discuss nonlinear optical response of such types of composite systems [7, 8, and 9]. In addition, for numerical calculations the sample with silver/dielectric composite with interfacial layer is utilized.

\section{Enhancement Factor of Local Field}

\subsection{Enhancement Factor of Local Field for Pure Metal Composite}

The distribution of the electric potential in the system is described by the following expressions

$$
\Phi_{m}=-E_{h} A r \cos \theta, r \leq r_{1}
$$




$$
\Phi_{h}=-E_{h}\left(r-\frac{B}{r^{2}}\right) \cos \theta, r \geq r_{1} .
$$

They are the solutions of the Laplace equations of the metal inclusion and the host matrix, respectively. Here $E_{h}$ is the applied field, $r$ and $\theta$ are the coordinates of the observation point (the beginning of the coordinate in the center of the inclusion and the $z$-axis is along $E_{h}$ ). We obtain a system of linear algebraic equations for unknown coefficients A and B from the continuity conditions of the potential and the displacement vector at the boundaries: metal-host matrix.

$$
\begin{aligned}
A & =\frac{3 \varepsilon_{h}}{2 \varepsilon_{h}+\varepsilon_{m}} \\
B & =\frac{\varepsilon_{m}-\varepsilon_{h}}{2 \varepsilon_{h}+\varepsilon_{m}} r_{1}^{3}
\end{aligned}
$$

The quantity $|A|^{2}$ which we call the enhancement factor can be presented as

$$
|A|^{2}=\frac{9 \varepsilon_{h}^{2}}{\left(2 \varepsilon_{h}+\varepsilon_{m}^{\prime}\right)^{2}+\varepsilon_{m}^{\prime \prime 2}}
$$

Where $\varepsilon_{h}$ is the dielectric function for the host matrix $\varepsilon_{m}^{\prime}$, $\varepsilon^{\prime \prime}{ }_{m}$ are the real and imaginary part of the metal dielectrics.

\subsection{Interfacial Layer Effect on Enhancement Factor of Local Field for Pure Metal Composite with Interfacial}

The distribution of the electric potential in the pure metal with the interfacial composite is described by the following expressions

$$
\begin{gathered}
\Phi_{m}=-E_{h} A r \cos \theta, r \leq r_{1} \\
\Phi_{s}=-E_{h}\left(B r-\frac{C}{r^{2}}\right) \cos \theta, r_{1} \leq r \leq r_{1}+t \\
\Phi_{h}=-E_{h}\left(r-\frac{D}{r^{2}}\right) \cos \theta, r \geq r_{1}+t
\end{gathered}
$$

They are the solutions of the Laplace equations of the metal inclusion, interfacial layer and the host matrix, respectively. We obtain a system of linear algebraic equations of unknown coefficients A, B, C, and D from the continuity conditions of the potential and the displacement vector at the boundaries: Metal-interfacial, interfacial-host matrix.

$$
\begin{gathered}
A=\frac{3 \varepsilon_{h}}{2 \varepsilon_{h}+\varepsilon_{m}+2 I / r_{1}} \\
B=\frac{2 \varepsilon_{h}}{2 \varepsilon_{h}+\varepsilon_{m}+2 I / r_{1}} \\
C=\frac{-\varepsilon_{h}}{2 \varepsilon_{h}+\varepsilon_{m}+2 I / r_{1}} r_{1}^{3} \\
D=\frac{\varepsilon_{m}+2 I / r_{1}-\varepsilon_{h}}{2 \varepsilon_{h}+\varepsilon_{m}+2 I / r_{1}} r_{1}^{3}
\end{gathered}
$$

The quantity $|A|^{2}$ which we call the enhancement factor can be presented as

$$
|A|^{2}=\frac{9 \varepsilon_{h}^{2}}{\left(2 \varepsilon_{h}+\varepsilon_{m}^{\prime}+{ }^{2 I} / r_{1}\right)^{2}+\varepsilon_{m}^{\prime \prime}}
$$

The real and the imaginary part of the metal dielectric represented using the Drude equations as

$$
\begin{gathered}
\varepsilon_{m}^{\prime}=\varepsilon_{\infty}-\frac{1}{z^{2}+\gamma^{2}} \\
\varepsilon_{m}^{\prime \prime}=\frac{\gamma}{z\left(z^{2}+\gamma^{2}\right)}
\end{gathered}
$$

The quantity shown in Eq. 13 is calculated by considering the limiting transition parameters. The limit, $t \rightarrow 0$ while $\varepsilon \rightarrow \infty$, the interfacial property is concentrated on a surface of zero thickness and only the quantity $t \varepsilon_{S}$ is of significance, we take

$$
I=\lim _{\substack{t \rightarrow 0 \\ \varepsilon \rightarrow \infty}} t \varepsilon_{S}
$$

To characterize the interface between pure metal particle and linear host matrix $I$, is just called the interfacial factor. The interfacial layer is, in fact, the mixture of metal and dielectric; since $\varepsilon_{S}$ is a complex number, $I$ is also a complex quantity. But, the real part of the dielectric function of metallic particle is always a large negative number, whereas the imaginary part is a small, positive one. Thus, for simplicity, in the limit case, we may neglect the imaginary part.

A maximum of the enhancement factor $|A|^{2}$ in this case is obtained by setting zero the first term in the denominator Eq. 13. It gives the linear equation with respect to $\varepsilon_{m}^{\prime}$.

$$
2 \varepsilon_{h}+\varepsilon_{m}^{\prime}+2 I / r_{1}=0
$$

We start our numerical calculations with the enhancement factor of a composite of spherical metal with interfacial inclusion in Fig. $1|A|^{2}$ versus $Z$ for different values interfacial factor $I=-2, I=0$, and $I=2$.

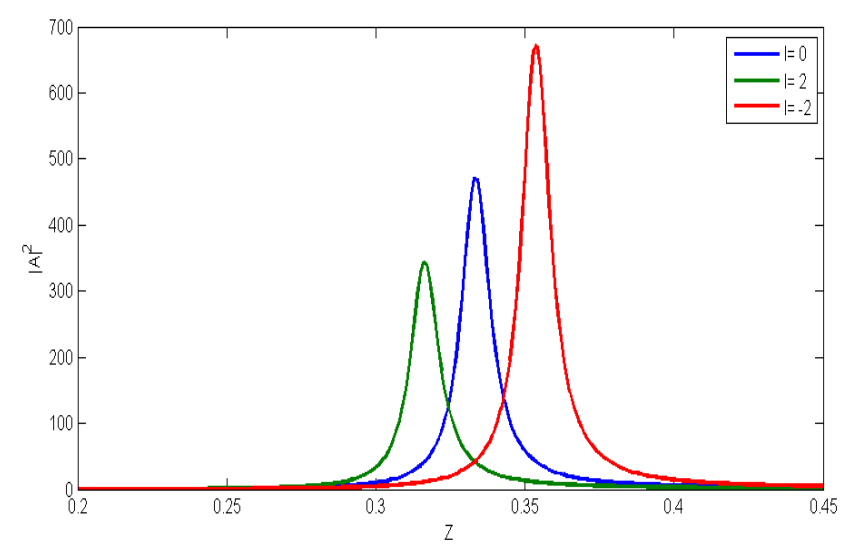

Figure 1. The enhancement factor $|A|^{2}$ for composites of small spherical silver metal nanoparticle embedded in dielectric host matrix versus $\mathrm{z}$. We use the following parameters of the system, $\gamma=1.15 \times$ $10^{-2}, \varepsilon_{\infty}=4.5, \varepsilon_{h}=2.25, I=-2,0,2$ 


\subsection{Interfacial Layer Effect on Enhancement Factor of Local Field for Spherical Metal/Dielectric Composite with Interfacial}

Let us consider an individual composite of small metal/dielectric separated by interfacial layer embedded in linear host matrix. The core is dielectric with the radius of $r_{1}$ and dielectric constant $\varepsilon_{d}$ depicted in metal of radius $r_{2}$ and dielectric of the metal $\varepsilon_{m}$. The interfacial layer considered in this work is between the dielectric core and metal. The interfacial layer dielectric constant is represented with $\varepsilon_{s}$ and radius of $r_{1}+t$ in small spherical metallic particle. In the electrostatic approximation; when a wavelength of the incident electromagnetic radiation is much greater than a typical size of the inclusion, the distribution of the electric potential in the system is described by the following expressions

$$
\begin{gathered}
\Phi_{d}=-E_{h} A r \cos \theta, r \leq r_{1}, \\
\Phi_{s}=-E_{h}\left(B r-\frac{C}{r^{2}}\right) \cos \theta, r_{1} \leq r \leq r_{1}+t, \\
\Phi_{m}=-E_{h}\left(D r-\frac{E}{r^{2}}\right) \cos \theta, r_{1}+t \leq r \leq r_{2} \\
\Phi_{h}=-E_{h}\left(r-\frac{F}{r^{2}}\right) \cos \theta, r \geq r_{2} .
\end{gathered}
$$

They are the solutions of the Laplace equations of the dielectric core, interfacial layer, metal inclusion and the host matrix, respectively. Here $E_{h}$ is the applied field, $r$ and $\theta$ are the coordinates of the observation point (the beginning of the coordinate in the center of the inclusion and the $Z$ axis is along $\left.E_{h}\right), \mathrm{A}, \mathrm{B}, \mathrm{C}, \mathrm{D}, \mathrm{E}$, and $\mathrm{F}$ are unknown coefficients.

From the continuity conditions of the potential and the displacement vector at the boundaries: Dielectric core-interfacial layer, interfacial layer-concentric metal shell and concentric metal shell-host matrix, we obtain a system of linear algebraic equations for A, B,C,D,E, and F given by Eq. 20 . Where $r_{1}, t$ and $r_{2}$ are the radii of the dielectric core, thickness of the interfacial layer and the radii of the metal shell of the inclusion, respectively. Further, we need only the coefficient $A$ that enters into the potential of the local field in the dielectric core. They can be presented in the form

$$
\begin{gathered}
A=\frac{9 \varepsilon_{h} \varepsilon_{m}}{2 p \Delta_{I}} \\
B=\frac{6 \varepsilon_{h} \varepsilon_{m}}{2 p \Delta_{I}} \\
C=\frac{-3 \varepsilon_{h} \varepsilon_{m}}{2 p \Delta_{I}} r_{1}^{3} \\
D=\frac{3 \varepsilon_{h}\left(\varepsilon_{d}+\frac{2 I}{r_{1}}+2 \varepsilon_{m}\right)}{2 p \Delta_{I}} \\
E=\frac{3 \varepsilon_{h}\left(\varepsilon_{d}+\frac{2 I}{r_{1}}-\varepsilon_{m}\right)}{2 p \Delta_{I}} r_{1}^{3}
\end{gathered}
$$

$$
F=\frac{\varepsilon_{m}\left[-\varepsilon_{h}\left(\frac{3}{p}-1\right)+2\left(\varepsilon_{d}+\frac{2 I}{r_{1}}\right)\left(\frac{3}{2 p}-1\right)\right]+\varepsilon_{m}^{2}-\varepsilon_{h}\left(\varepsilon_{d}+\frac{2 I}{r_{1}}\right)}{2 \Delta_{I}} r_{2}^{2}
$$

Where

$$
\Delta_{I}=\varepsilon_{m}^{2}+Q \varepsilon_{m}+\left(\varepsilon_{d}+\frac{2 I}{r_{1}}\right) \varepsilon_{h}
$$

Here

$$
Q=\left(\varepsilon_{d}+\frac{2 I}{r_{1}}\right)(3 / 2 p-1)+\varepsilon_{h}(3 / p-1)
$$

Where $p=1-\left(r_{1} / r_{2}\right)^{3}$ is a metal fraction in the inclusion, $\varepsilon_{d}, \varepsilon_{s}, \varepsilon_{m}$ and $\varepsilon_{h}$ are the dielectric functions of the dielectric core, interfacial layer, metal shell and the host matrix, respectively. We note that the expressions 22 have been used in [10] while studying the optical induced bistability in dielectric matrix with spherical metal inclusions with small nonlinear dielectric core. With interfacial layer; the expression for Eq. 22 gives the corresponding result of [1] for the polarizability of a coated sphere. The local field $\mathrm{E}$ in the dielectric core can be obtained with the help of relation

$$
E=|A| E_{h}
$$

Where $|\mathrm{A}|$ is given by Eq. 22 in general, it is a complex function. Further, it would be convenient to deal with the real quantity $|A|^{2}$, which we call the enhancement factor. It can be presented as follows

$$
|A|^{2}=\frac{81}{4 p^{2}} \frac{\varepsilon_{h}^{2}\left(\varepsilon_{m}^{\prime}{ }^{2}+\varepsilon_{m}^{\prime \prime 2}\right)}{\left(\left(\varepsilon_{m}^{\prime 2}-\varepsilon_{m}^{\prime \prime 2}+Q \varepsilon_{m}^{\prime}+\left(\varepsilon_{d}+\frac{2 I}{r_{1}}\right) \varepsilon_{h}\right)^{2}+\varepsilon_{m}^{\prime 2}\left(Q+2 \varepsilon_{m}^{\prime}\right)^{2}\right)}
$$

Eq. 31 is calculated by considering the limiting transition parameters mentioned in Eq. 16. To characterize the interface between dielectric core and the concentric metallic particles $I$ is just called the interfacial factor. The interfacial layer is, in fact, the mixture of metal and dielectric; since $\varepsilon_{S}$ is a complex number, $I$ is also a complexquantity. But, the real part of the dielectric function of metallic particle is always a large negative number, whereas the imaginary part is a small, positive one. Thus, for simplicity, in the limit case, we may neglect the imaginary part.

The dielectric function of the dielectric core and the dielectric function for the host are taken to be linear for the sake of simplicity. That means we will ignore the imaginary parts of $\varepsilon_{d}$ and $\varepsilon_{h}$. The analytical calculation is considered with $\gamma$ negligible. The maximum of the enhancement factor in this case is obtained setting zero the first term in the denominator Eq. 31. It gives the quadratic equation with respect to $\varepsilon_{m}^{\prime}$

$$
\varepsilon_{m}^{\prime 2}+Q \varepsilon_{m}^{\prime}+\left(\varepsilon_{d}+\frac{2 I}{r_{1}}\right) \varepsilon_{h}=0
$$

The numerical calculation for the enhancement factor of the local field of composites of small spherical metal/dielectric inclusion with interfacial layer is 


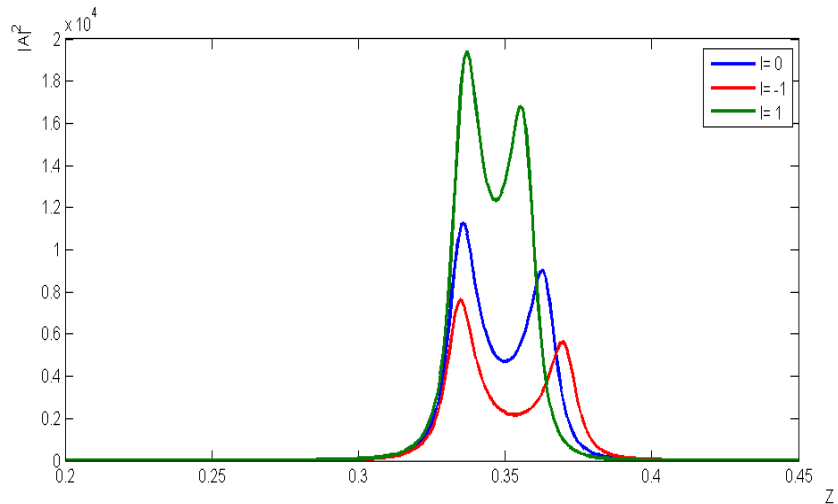

Figure 2. The enhancement factor $|A|^{2}$ for composites of small spherical silver metal/Dielectric nanoparticle separated by Interfacial layer embedded in dielectric host matrix versus $\mathrm{z}$. We use the following parameters of the system: $\gamma=1.15 \times 10^{-2}, \varepsilon_{\infty}=4.5, \varepsilon_{h}=2.25, \varepsilon_{d}=6$, $I=-1,0,1$

In Fig.2, we present $|A|^{2}$ of composites of small spherical metal/dielectric separated by interfacial layer verses the resonant frequency $Z$ for three different interfacial layer values; $I=-1, I=0$ and $I=1$. We find that the interfacial layer plays an important role in enhancement factor behavior. It is shown that with the change of $I$ from negative value to positive value, namely, with the transition of the interfacial layer from dielectric property to metallic property, the threshold values of the enhancement factor of the local field increase, since the system is considered the outer part is metal that is why the enhancement increases towards the metallic character. Furthermore, comparing with the case of no interfacial layer, the metal-like interfacial layer makes the threshold values increase, while the dielectric like interfacial layer makes the threshold values decrease.

\section{Interfacial Layer Effect on the Optical Induced Bistability for Metal/Dielectric Composite}

The dielectric function for a metal in the inclusion is given to be in the Drude form Eq. 14 and 15. The DF of the core $\varepsilon_{d}$, in general case, includes a nonlinear part with respect to the local field.

$$
\varepsilon_{d}=\varepsilon_{d 0}+\chi|E|^{2}
$$

Where $\varepsilon_{d 0}$ is the linear part of DF and $\chi$ is the nonlinear Kerr coefficient; $\boldsymbol{E}$ is thelocal field in the core. The enhancement factor of the local field in the inclusion for the weak incident fields $\chi|E|^{2} \ll \varepsilon_{d 0}$, the local field is presented as in the form of equation $E=|A| E_{h}$. In this relation $|\mathrm{A}|$ is given by Eq. 22 which is a complex quantity. Further, it would be convenient to consider with $|A|^{2}$, whichrepresent a real quantity. We call $|A|^{2}$ the enhancement factor and express it as Eq. 31. Here $\varepsilon_{m}^{\prime}$ and $\varepsilon_{m}^{\prime \prime}$ are the real and imaginary part of $\varepsilon_{m}$ given by Eq. 14 and 15 , respectively. For the sake of simplicity, we ignore the imaginary parts of $\varepsilon_{h}$.

From $E=|A| E_{h}$ we obtain the cubic equation for the square modulus of the local field, $X=\chi|E|^{2}, Y=$ $\chi\left|E_{h}\right|^{2}$ in the form

Where

$$
\alpha X^{3}+\beta X^{2}+\delta X=\eta Y
$$

$$
\begin{gathered}
\alpha=d_{2}^{2}+d_{4}^{2} \\
\beta=2\left(d_{1} d_{2}+d_{3} d_{4}\right) \\
Y=\chi\left|E_{h}\right|^{2} \\
\delta=d_{1}^{2}+d_{3}^{2} \\
\eta=\frac{81 \varepsilon_{h}^{2}\left(\varepsilon_{m}^{\prime}{ }^{2}+\varepsilon_{m}^{\prime \prime}\right)}{4 p^{2}}
\end{gathered}
$$

With $a=3 / p-1, b=3 / 2 p-1, c=\varepsilon_{d 0}+2 I / r_{1} d_{1}=$ $\varepsilon_{m}^{\prime} \varepsilon_{h} a+\varepsilon_{m}^{\prime} b c+\varepsilon_{m}^{\prime 2}-\varepsilon_{m}^{\prime \prime 2}+\varepsilon_{h} c, d_{2}=\varepsilon_{m}^{\prime} b+\varepsilon_{h}$ ,$d_{3}=\varepsilon_{m}^{\prime \prime}\left(\varepsilon_{h} a+b c+2 \varepsilon_{m}^{\prime}\right)$ and $d_{4}=\varepsilon_{m}^{\prime \prime} b$.

The quantity $X$ dependson the applied field $E_{h}$ and the parameters of the composite. If the cubic equation Eq. 34 has one real positive root, the local field in the nano-particle is a single-valued function of the applied field. If it has three real positive roots, the local field is not a single-valued function of the applied field and the system becomes unstable. This is what we called optical induced bistability (OIB) [11]. OIB is usually illustrated in the $\mathrm{Y}-\mathrm{X}$ plane and connected with S-like curves showing that single value of $\left|E_{h}\right|^{2}$ is able to activate three different values of the local field $|E|^{2}$. This phenomenon is called the Induced Optical Bistability $[1,12]$.

Now, I is only a real number. When I is taken as a negative (or positive) value, the interface exhibits dielectric-like (or metal-like); and I = 0 corresponds to no interface. Numerical results for three different interfacial layers $\mathrm{I}=-2,0$ and 2 by 3.1.1 and MatLab software version 7.10.0.499 (R2010a) codes we obtain the following Fig 3. Interfacial effect plays an important role in a variety of systems, and it can dramatically alter the systems' optical behavior and optical bistability. In this paper, the effect of interfacial layer on the optical bistability is qualitatively studied; by introducing the interfacial factor $I$.

This plot Fig 3 exhibits bistable response clearly, and the parts of the curves with negative slope are unstable. We find that the interfacial layer plays an important role in bistable behavior. It is shown that with the change of $I$ from positive value to negative value, namely, with the transition of the interfacial layer from metallic property to dielectric property, the threshold values of the bistability decrease. Compared with the case of no interfacial layer, the metal-like interfacial layer makes the threshold values increase, while the dielectric-like interfacial layer makes the threshold values decrease. With increasing $I$, both the threshold values and bistable region are increasing, and we have known that the threshold values will increase for the case of metal-like interfacial layer; hence,the threshold values will increase. 


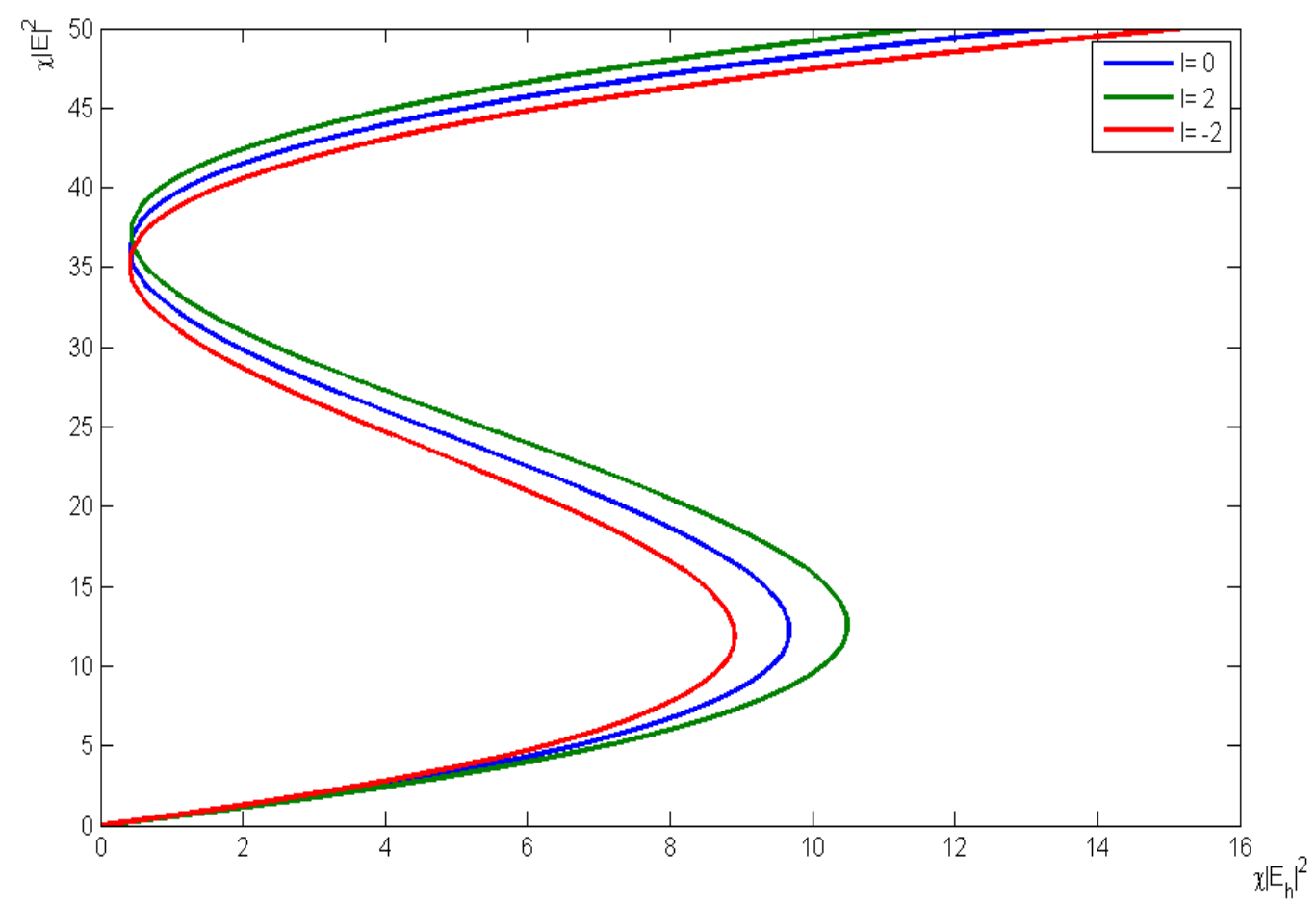

Figure 3. Optical Induced Bistability (IOB)in composites of small spherical metal/dielectric separated by interfacial layer in linear host matrix $\varepsilon_{d 0}=6$, $\gamma=1.15 \times 10^{-2}, \varepsilon_{\infty}=4.5, \varepsilon_{h}=2.25, p=0.99$

\section{Conclusions and Recommendation}

In this paper, we studied the effect of interfacial layers on the enhancement factor of local field and optical induced bistability for small spherical metal/dielectric composite randomly embedded in linear host matrix. We have solved the enhancement factor of local field for metal/dielectric composites with interfacial embedded in a linear host matrix and Optical Induced Bistability (OIB) metal/dielectric composites with interfacial embedded in a linear host matrix. We have solved Laplace equation with Drude model together with Taylor. In this we have found several interesting phenomena which are related with the enhancement factor and Optical Induced Bistability. Furthermore, we noted interfacial layer $I$ with thickness $t$ and dielectric function $\varepsilon_{s}$ in between dielectric core and concentric metal shell, the enhancement factor of a composites of small spherical metal/dielectric composite with Interfacial is extremely increased, meanwhile the corresponding peak is enhanced when the interfacial layer is changed from dielectric-like to metal-like, the peak corresponding to metal-like interfacial layer can be enhanced strongly.

Generally the analytical and numerical results show that the interfacial layer can greatly affect the Enhancement Factor of the local field and Optical Induced Bistability in metal inclusions with small dielectric cores, and that the metal-like interfacial layer is favorable to increase the threshold value of the OIB domain.

\section{Acknowledgements}

I would like to acknowledge to Jimma University and Wolkite University funding the project.

\section{REFERENCES}

[1] C.F. Bohren and D.R.Huffman, Absorption and scattering of light by small particles, Wiley, New York, 1983.

[2] U. Kreibig and M. Vollmer, Optical properties of metal clusters, Springer, Berlin,1995.

[3] V. M. Shalaev, Electromagnetic properties of small-particle composites, Phys. Reports 61 (1996), 272.

[4] K.M. Leung, Optical bistability in the scattering and absorption of light from nonlinear microparticles, Phys. Rev. A 33 (1986), 2461.

[5] D. Stroud D.J.Bergman, Ohad Levy, Theory of optical bistability in weakly nonlinear composite medium, 49 (1994), 129-134.

[6] R. Nathanshon Levy and D. J. Bergman, Electrical resonance and optical bistability in periodic composite materials, J. Appl. Phys 77 (1995), 4263-4273.

[7] L. GAO and J. Z.Y. LI, Appl. Phys. 87 (2000), 1620.

[8] L. GAO J.P. HUANG and Z.Y. LI, Solid state, Commun. 115 (2000), 347.

[9] S. Torquato and M.D. Rintoul, Phys. Rev. Lett. 75 (1995), 4067. 
[10] V.N. Mal'nev Sisay Shewamare, Two optical bistability domains in composites of metal nanoparticles with nonlinear dielectric core, Physica B 407 (2012), 4837- 4842.

[11] Nonlinear optics, Academic Press, 1992.
[12] R. Inguva M.H. Birnboim N. Kalyaniwalla, J.W. Haus, Intrinsic optical bistability for coated spheroidal particles, Phys. Rev. A 42 (1990), 5613. 